\title{
Comparison of accelerometer-based energy expenditure estimation algorithms
}

\author{
Niall Twomey, Dr. Stephen Faul, Dr. William P. Marnane \\ Dept. of Electrical and Electronic Engineering \\ University College Cork \\ Cork \\ Email: niallt@rennes.ucc.ie
}

\begin{abstract}
Many accelerometer-based energy expenditure estimation algorithms and platforms have been established in recent topical literature, and each boasts a high correlation against the gold standard in energy expenditure measurement, i.e. indirect calorimetry. The aim of this study was to implement a set of these algorithms, run them all over a common dataset and investigate the strengths and weaknesses associated with each. The algorithms were then ported to a SHIMMER device for a real time, mobile and non-invasive energy expenditure estimation solution. High correlations were found between the accelerometer-regressed energy expenditure estimates and the reference dataset both on a computer and SHIMMERimplementation of the algorithms.
\end{abstract}

Keywords-Energy expenditure estimation, accelerometers, indirect calorimetry.

\section{INTRODUCTION}

There exists no reliable, non-invasive, portable and widely validated energy expenditure measurement sensor. When one considers that statistics report that a large percentage of today's youth and adults as being overweight and that a large portion of our elderly suffer from conditions that inhibit their physical activity the need for a reliable, objective and portable energy measurement system is self evident. While comparisons have been drawn between different energy expenditure platforms[1] the authors believe that algorithmic comparisons have not been investigated.

Indirect calorimetry is the gold standard in energy expenditure measurement and was the means employed to record reference energy expenditure levels in this study. The heat that a living organism produces is a function of the ratio of $\mathrm{O}_{2}$ and $\mathrm{CO}_{2}$ it expresses, and indirect calorimetry exploits this fact to regress the heat energy to an energy expenditure measurement, i.e. kilo calories, kilo Joules etc. The calorimeter that was used was the CPET (Cardio Pulminary Exercise Testing) metabolic cart[2].

To measure the gaseous composition of the breath a gas mask is placed over the mouth and nose of the subject. A hose is attached to the gas mask and this leads to the indirect calorimeter. A good seal between the gas mask and the face must be present to ensure that the gasses that are analysed by the calorimeter have solely been sourced from the breath.

Energy expenditure can be quantified in many different ways. The most commonly used energy measurement units

Permission to make digital or hard copies of all or part of this work for personal or classroom use is

granted without fee provided that copies are not made or distributed for profit or commercial advantage

and that copies bear this notice and the full citation on the first page. To copy otherwise, to republish,

to post on servers or to redistribute to lists, requires prior specific permission and/or a fee.

PERVASIVEHEALTH 2010, March 22-25, Munchen, Germany

Copyright $\odot 2010$ ICST 978-963-9799-89-9

DOI 10.4108/ICST.PERVASIVEHEALTH2010.8879
Table I

MET ACTIVITY INTENSITY CLASSIFICATION

\begin{tabular}{cc} 
Activity level & MET range \\
\hline \hline Light activity & Under 3 \\
Moderate activity & Between 3 and 6 \\
Vigorous activity & Above 6
\end{tabular}

in literature are kilo-calories/minute, kilo-Joules/minute. However, other lesser known units, such as metabolic equivalents (METs), exist and are sometimes used as the unit of regression in energy expenditure estimation. A MET is defined as $3.5 \mathrm{ml} \mathrm{O}_{2} / \mathrm{kg} / \mathrm{min}$, and is commonly used in the quantification of the extent of activity intensity, see Table I.

If, while being monitored by the CPET, one was exercising, the metabolic rate of the subject will change according to the extent of the exercise. The response, however, is not instantaneous, and is similar to the charging of a capacitor in a resistor/capacitor network in response to a step input.

A SHIMMER (Sensing Health with Intelligence, Modularity, Mobility, and Experimental Reusability) mote is a wireless sensor platform that is geared towards wearable applications[3]. The SHIMMER has a small form-factor $(50 \mathrm{~mm} \times 25 \mathrm{~mm} \times 12.5 \mathrm{~mm})$, is very light (15 grammes) and can sample three channels of 12-bit ADC at $50 \mathrm{~Hz}$ for up to ten days.

Recent researcher in activity monitoring have published exciting results in this form of energy expenditure estimation[4]. The favoured means of measuring this is with microelectromechanical systems (MEMS) accelerometers. Accelerometers are sensors which can detect acceleration along a directional axis. If three of these are orthogonally orientated then three dimensional accelerations can be measured. Accelerometer based expenditure estimation initially used uniaxial accelerometers, as these were the first MEMS accelerometers to be fabricated. Triaxial accelerometers were then introduced as three uniaxial accelerometers orthoganally mounted on a cube, until triaxial accelerometers were fabricated on one chip.

Onboard a SHIMMER device is a microprocessor (MSP430), Bluetooth radio (Mitsumi WML-C46N CSR based design), micro SD card slot, a triaxial accelerometer (Freescale MMA7260Q 1.5/2/4/6g Micropower MEMs 
Table II

PHYSICAL CHARACTERISTICS OF VOLUNTEERS FOR THE STUDY

\begin{tabular}{cccc} 
ID & Age $(\mathrm{yr})$ & Height $(\mathrm{cm})$ & Weight $(\mathrm{kg})$ \\
\hline \hline 1 & 23 & 174.5 & 77.5 \\
2 & 25 & 183 & 81.8 \\
3 & 22 & 187 & 86.7 \\
4 & 24 & 179 & 87 \\
5 & 30 & 170 & 88 \\
\hline Mean \pm SD & $24.8 \pm 3.1$ & $178.7 \pm 6.72$ & $84.2 \pm 4.45$
\end{tabular}

Accelerometer) as well as other electronic components. The range of applications that the SHIMMER can be used for is extensive. This is due to the fact that the firmware of the microprocessor can be programmed by a suitable user to perform specific task. The devices are programmed in a language called nesC (network embedded systems C) and the microprocessor is embedded with the TinyOS component based operating system.

It is with the SHIMMER that the comparison of energy expenditure estimation for this study is to be accomplished. SHIMMERs were strapped to different locations on the body as a volunteer walks on a treadmill. As they exercise the measured accelerations are streamed in real time via the Bluetooth data link and the accelerations are stored on a computer for analysis.

\section{Methods}

\section{A. Experimental setup}

Five healthy male subjects participated in this study. The physical characteristics of each subject were recorded and a summary can be seen in Table II.

A treadmill (Powerjog GX100) was set up to operate from $3 \mathrm{kmph}$ to $7 \mathrm{kmph}$ at $1 \mathrm{kmph}$ increments. The subjects were requested to walk on it at these speeds and five accelerometers were placed on the body to record acceleration data. The accelerometers were placed on the ankle, knee, waist, wrist and arm and were attached to the body via custom-made neoprene straps. All the accelerometers were positioned on the right side of the body as it was reasoned that over an epoch the accelerations on one side of the body should be similar to those measured on the other side of the body. This will be discussed in Section III-A.

The treadmill ran at each speed for four minutes at a $1 \%$ gradient, to emulate walking on a flat surface[5]. The time for which the subject walked at each speed was chosen at four minutes due to [6] and [7]. Reference [6] provides the upper bound to the amount of time that an exercise must be performed for the body to have reached a steady metabolic state as between $2-3$ minutes, depending on the fitness of the individual. Reference [7] limits the upper time limit on the routine of this study for a reliable metabolic response at twenty, as the body is exerted to more physical exertion different attributes of the body will change the metabolic rate of the process.

A gas mask was fixed over the subject's mouth and nose and the net was tightly fastened around the subject's head. A hose leaves the mask and is attached directly to the gas analysers in the calorimeter. To ensure that no gas entered or exited the seal about the subject's face, other than that contributed by breathing, the subject was asked to momentarily block the outlet of the gas mask and if they were unable to express air during this very brief time the mask was deemed securely fastened.

Authors in this area typically sample the accelerations at moderately low sampling frequencies $-50 \mathrm{~Hz}$ is common, but other frequencies are also seen in literature. In order to allow the database of accelerations that have been recorded to be used over a wide range of algorithms (not necessarily only those which sample at $50 \mathrm{~Hz}$ ) accelerations were sampled at a higher sampling rate of $250 \mathrm{~Hz}$. This will allow a resampling algorithm, when required, to downsample to the required sampling rate with a high confidence in the accuracy of the new dataset. According to [4], many papers cite the fact that the body cannot be expected to generate motion that is quicker than $15-20 \mathrm{~Hz}$ so signals above these frequencies are filtered out.

The accelerometers on the ankle, knee, wrist and arm recorded acceleration only, but the accelerometer on the waist also recorded the electrocardiogram (ECG) through an ECG daughterboard and on the SHIMMER and ECG electrodes that were affixed to the body in an Einthoven triangle configuration. The ECG signal was also sampled at $250 \mathrm{~Hz}$. The purpose of this is to investigate the effects of marrying different types of energy expenditure algorithms together to one algorithm, i.e. acceleration and heart rate, acceleration and heart rate variability etc. A reference heart rate was provided by a polar chest heart rate strap (S610i). The acceleration and ECG data was transmitted via Bluetooth radio from the SHIMMER devices to a nearby PC where the data packets from each SHIMMER were parsed by a custom written program, written in $\mathrm{C}++$, and stored to a file.

\section{B. Analysis}

1) Symmetry verification: To verify the assumption of symmetry between accelerations on the right and left hand sides of the body a test was set up whereby accelerometers were placed on the right and left wrists and ankles and while a subject walked the accelerations on these parts of the body were recorded, stored in file and analysed.

2) Codeword to acceleration conversion: The microprocessor on the SHIMMERs used in this study sample raw output values from accelerometers. These values are then converted to a digital codeword by the microprocessor's ADC module. In order to convert the codeword to an acceleration value (of dimensions $m s^{-} 2$ or multiples of $g$ ) 


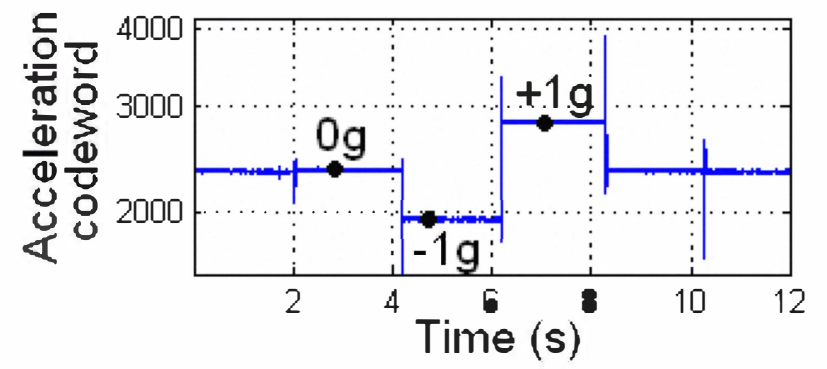

Figure 1. Codeword values at adjacent device orientations

an offset must be subtracted from the codeword (to centralise it from the $0 g$ position) and this result must then be scaled by a constant value to complete the conversion. As each device is unique these scaling values vary from device to device so an efficient search algorithm was required to discover these values for each SHIMMER used.

As a result of the orthogonality of the accelerometer's axes (1) is true for stationary devices due to the device experiencing Earth's gravitational field.

$$
\sqrt{\sum_{i=x}^{z}\left(\frac{a_{i d}-d i_{0 g}}{d i_{1 g}}\right)^{2}}=1 g=9.81 m s^{-2}
$$

Where $i$ is the axis that is being considered $a_{i d}$ are the digital codewords of the accelerations sensed in the $i^{\text {th }}$ direction (i.e. $\mathrm{x}, \mathrm{y}$ or $\mathrm{z}$ ), $d_{i_{0 g}}$ are the offsets that when subtracted from the measured value scale the acceleration from the $0 g$ point, and $d_{i_{1 g}}$ are the values that scale the digital representation of the acceleration value to g's, i.e. the digital $1 \mathrm{~g}, 9.81 \mathrm{~ms}^{-2}$ value.

To find these points the triaxial accelerometer is orientated for a given time period on its six faces. If the surface the accelerometer was being placed on was perfectly level and the faces of the accelerometer were completely orthogonal the average between two sets of readings, for one face and its opposite counterpart, would provide the $0 g$ position for that axis. The distance between midpoint and the extremities would then provide the $1 g$ value for the axis, see Figure 1.

However, these conditions cannot be assumed true. The points that convert the values recorded on each axis can be realised by finding a point in the range $[0,4095]$ on each axis on the accelerometer, resulting in $4096^{3}$ possible solutions in total.

A brute force search for this value is not feasible, so a 3D binary search algorithm was implemented to search for the values that minimise the standard deviation of the magnitude of the instantaneous accelerations in (1). Depending on the maximum and minimum values recorded on different orientations requiring the number of iterations given by (2), yielding $12^{3}$ iterations at worst.

$$
N=2^{\left(\operatorname{ceil}\left(\log _{2}(\max \text { Value }-\operatorname{minValue})\right)\right)^{3}}
$$

The 'ceil' function rounds a number up to the next highest integer. Using this method the codewords received are scaled to provide accurate acceleration measurements of the accelerations the devices experienced.

3) Breath and acceleration synchronisation: The energy expenditure levels that were measured by the CPET and saved to a computer were recorded on a breath-by-breath basis. The breathing rate of a human is not periodic, especially over a range of exercises, so the dataset that is used as the reference for the accelerometer-regressed energy expenditure levels is not regularly distributed over time. The time-tag associated with the measurement of each breath is also rounded to the nearest whole second, introducing further inaccuracies.

For comparison purposes the reference data must be on a common time-base with the regressed data, i.e. a reference energy expenditure value must be available every regression epoch. The time base that the reference data must match was given by the epoch time, $T_{e}$, of the algorithms that are implemented, discussed later. Two means of achieving the time correlation were investigated.

The first method involved casting a cubic spline curve interpolation over the reference dataset (the energy expenditure values and the times associated with each of these were passed as spline parameters). The value of the spline curve at each integer multiple of $T_{e}$ was taken as the energy expenditure reference for that epoch.

For the range of time that an epoch exists a number of breaths will occur. The second method that was implemented averages the energy expenditure levels within the range of the epoch, $[t, t+T e)$, and uses this as the energy expenditure level for the current epoch. If no breaths occur, which is unlikely, 0 is returned, as no energy expenditure was detected; this is not guaranteed with the splining method.

4) Algorithm implementation: Four algorithms were investigated in this study: one by Bouten[8], two by Chen[9] and one by Crouter[10].

Bouten[8] collected a large set of triaxial acceleration and indirect calorimetry energy expenditure data. With the dataset and knowledge of conditions provoking the accelerations was able to formulate an acceleration-based energy expenditure estimation algorithm and published (3).

$$
\mathrm{EE}_{\text {act }}=0.104+0.023 \times \mathrm{IAA}_{\text {tot }}
$$

Where $\mathrm{EE}_{\text {act }}$ is the energy expenditure estimation due to activity that the equation calculates and $I A A_{\text {tot }}$ is a dimensionless unit known as the integral of the absolute value of the acceleration, see (4). 


$$
\mathrm{IAA}=\int_{t=\tau}^{\tau+T_{e}}\left(\left|a_{x}\right|+\left|a_{y}\right|+\left|a_{z}\right|\right) d t
$$

Bouten et al. collected a large set of acceleration and reference calorimeter data. Linear regression was performed over the database to convert the acceleration values to energy expenditure measurements resulting to the parameters of (3).

Chen's algorithms[9] use a waist mounted accelerometer and the approach was to first separate the horizontal ( $x, y)$ and vertical $(\mathrm{z})$ acceleration components that were detected by the accelerometers. The accelerations were converted to vertical and horizontal acceleration counts for the epoch being studied (horizontal component $H(k)=\sqrt{a_{x}(k)^{2}+a_{y}(k)^{2}}$, and vertical component $\left.V(k)=\sqrt{a_{z}(k)^{2}}\right)$. These acceleration counts were then combined with subject-specific linear parameters and the acceleration-based EE estimate resulted. Two algorithms are presented, the first, linear algorithm[9], is summarised in by (5).

$$
\begin{aligned}
a_{L}= & (5.76 \times \operatorname{mass}(\mathrm{kg})+ \\
& 11.95 \times \operatorname{height}(\mathrm{cm})+ \\
& 6.89 \times \operatorname{age}(\mathrm{yr})-2,001) / 1000 \\
b_{L}= & \frac{5.96 \times \operatorname{mass}(\mathrm{kg})+349.5}{1000} \\
E E_{a c t}(k)= & a_{L} \times H(k)+b_{L} \times V(k)
\end{aligned}
$$

Chen's second algorithm[9] is similar to the first, linear, algorithm. Much of the research in accelerometer-based regression is based about generating linear regression equations, as seen in (5), but it is not clear if the regression should be linear. A nonlinear regression equation was also computed by Chen to investigate this point, and the equations used by this algorithm are given by (6).

$$
\begin{aligned}
& p 1= \frac{2.66 \times \operatorname{mass}(\mathrm{kg})+146.72}{1000} \\
& p 2= \frac{-3.85 \times \operatorname{mass}(\mathrm{kg})+968.28}{1000} \\
& a_{N}= \frac{12.81 \times \operatorname{mass}(\mathrm{kg})+843.22}{1000} \\
& b_{N}=(38.90 \times \operatorname{mass}(\mathrm{kg})- \\
& 682.44 \times \operatorname{gender}+ \\
&692.44) / 1000 \\
& E E_{a c t}(k)=a_{N} \times H(k)^{p 1}+b_{N} \times V(k)^{p 2}
\end{aligned}
$$

Where gender is defined as 1 for men, and 2 for women. These algorithms were chosen because the parameters of the

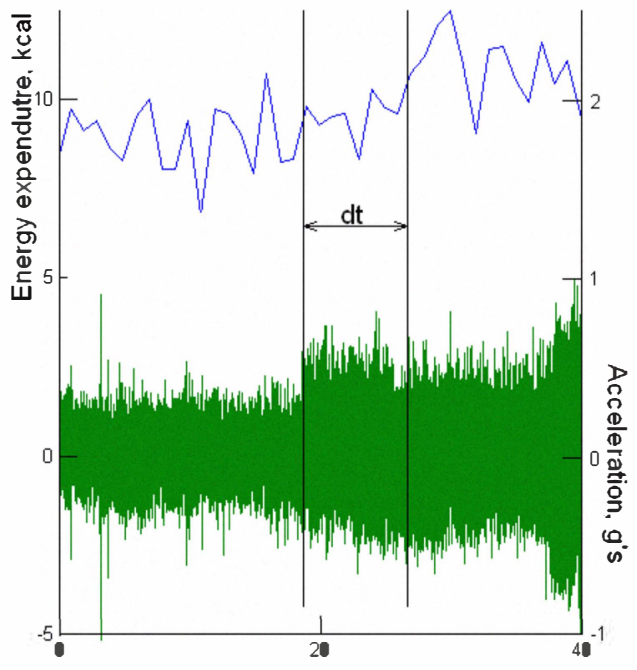

Figure 2. Time lag between speed change and energy expenditure, $d t$

equations are subject-specific, i.e. they are a function of the physical characteristics of the person, and to investigate the benefits/drawbacks of linear and nonlinear subject-specific regression parameters.

Similar to Bouten et al., Chen's algorithms resulted from both linear and nonlinear regression on the dataset that was collected. This regression differed due to the introduction of the physical characteristics of the subjects into the regression algorithms and the format of the resulting equations (that the parameters were themselves parametrised).

The final algorithm that was studied was Crouter's algorithm[10]. When the instantaneous acceleration captured by the devices is plotted against the energy expenditure measured by the CPET, a time lag can be seen between the accelerations detected at the step change in the speed of the treadmill and the metabolic response, see Figure 2. The output of the previous algorithms would instantaneously change once the speed of the treadmill has been increased as they are functions of the acceleration that was recorded over the last epoch. Crouter's algorithm attempts to follow the trend of the metabolic response with a regression equation that is a function of the previous minute's worth of epoch readings.

Where the Bouten's and Crouter's algorithms provide one equation that is called at the end of each epoch Crouter's algorithm uses a decision tree to determine which of the equations he generated to use[10].

5) SHIMMER-domain implementation: The SHIMMER was used for data aggregation, but it was also tested for applicability as a mobile, reliable and non-invasive energy expenditure measurement platform. The algorithms from Section II-B4 were implemented on the SHIMMER and a comparison between the software results and the SHIMMER-domain results was performed. 
Table III

PERCENTAGE DIFFERENCE IN RIGHT AND LEFT BODY ACCELERATIONS, $\mathrm{N}=7$

\begin{tabular}{ccccc} 
Location & Mean & S.D. & Max & Min \\
\hline \hline Wrist & $0.7879 \%$ & $0.4409 \%$ & $1.5848 \%$ & $0.0206 \%$ \\
Ankle & $1.7763 \%$ & $0.7174 \%$ & $2.9969 \%$ & $0.6343 \%$
\end{tabular}
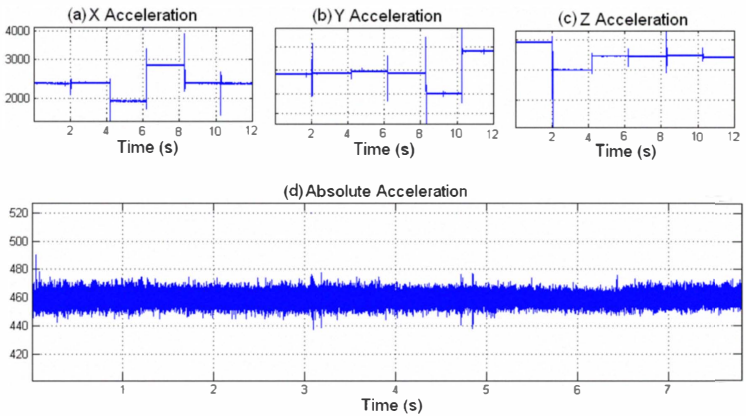

Figure 3. Individual and combined acceleration trace for $0 \mathrm{~g}$ offset test

\section{RESULTS}

\section{A. Symmetry verification}

After the collection of body accelerations for the symmetry test the accelerations that were recorded on the right and left hand side were analysed. The IAA, see (4), of the acceleration vector was used over a 10 second epoch and the results on the right and left sides of the body were compared against one another. Table III is a summary of the results found from the experiment. It was found that the difference in IAA between the right and left sides of the body differed by no more than $3 \%$. This difference was deemed small and it was decided to use the accelerations on the right hand side of the body on every subject accordingly.

\section{B. Codeword to acceleration conversion}

Figure 3 is a trace of the accelerations measured when the SHIMMER devices were orientated on their six faces, Figure $3(a, b, c)$, and the magnitude combination when the offset that provided the smallest standard deviation from the mean was discovered, Figure 3(d), which, due to (1), is expected to be a constant value equalling $1 g$. As the experiment was set up for orientation analysis the SHIMMER device was stationary on each axis for a time period until it was flipped to the other axes. Equal contributions of constant, non-switching, accelerations were used in order that each axis factored equally to the resulting offsets, i.e. the disturbances seen in Figures 3(a,b,c) were omitted from the calculations.

When the results from the binary search were employed and the standard deviation of magnitude of the acceleration were subsequently inspected the mean standard deviation (over all the SHIMMERs' accelerometers, $N=5$ ) was calculated as $1 g \pm 0.0082 g$ (mean \pm SD) for static devices, $1 g$ due to (1). The small standard deviation from the mean

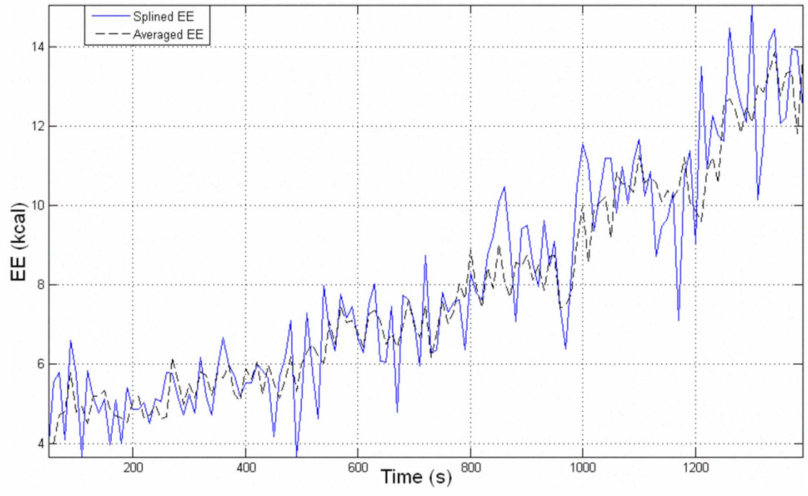

Figure 4. Averaged and splined energy expenditure plotted against time

suggests that the searching algorithm functions well for the accelerometers that were studied.

\section{Breath and acceleration synchronisation}

1) Reference energy level: Figure 4 plots the energy expenditure values of the splined and averaged reference energy expenditure against a common time base. As expected both curves follow the same trend. In general the variation in the splined curve is higher than that of the averaged curve. This is to be expected as in generating the values for the averaged dataset the algorithm averages a number of data points over the epoch range, introducing a filtering effect.

It was decided to use the results of the averaging method as the reference against which the accelerometer-based energy expenditure estimates will be compared. This was chosen because the times that were associated with each breath were rounded to whole second numbers. The spline curve fitting function requires data points coupled with associated time stamps. If confidence cannot be found in the time stamp one cannot be assured that the curve returned is accurate. This is not an issue for the averaging algorithm as it depends on values found within the range of an epoch and is invariant to the exact time the value was recorded.

2) Energy expenditure normalisation: Crouter's algorithm regresses to a unit of energy expenditure measurement called METs (metabolic equivalent). This unit is defined as $3.5 \mathrm{ml} \mathrm{O} \mathrm{Kg}^{-1} \mathrm{~min}^{-1}$ so is a function of the energy expenditure and the mass of the subject in question. As neither Chen nor Bouten regressed to this unit of energy it was necessary to scale the reference and the regressed energy values to a common standard so that all algorithms can be compared on the same grounding. This was accomplished by (7).

$$
\hat{f}=\frac{f-\min (\text { ref })}{\max (\text { ref }-\min (\text { ref }))}
$$

Where $f$ is the signal that's to be scaled (can either be the reference or regressed datasets), ref is the reference data (i.e. the dataset recorded by the $C P E T$ ), and $\hat{f}$ is 
Table IV

TABULATION OF RMSE VALUES FOR EACH ALGORITHM, OVER EVERY VOLUNTEER FOR DATA COLLECTION, $N=7$

\begin{tabular}{cc} 
Algorithm & RMSE \pm SD \\
\hline \hline Chen nonlinear & $0.065737 \pm 0.003285$ \\
Chen linear & $0.080713 \pm 0.013166$ \\
Bouten & $0.075382 \pm 0.019273$ \\
Crouter & $0.135009 \pm 0.014078$
\end{tabular}

the scaled reference/dataset. This function is called on the reference data, to scale it between the range of 0 and 1 , and the regressed dataset. Figure 5 shows the plots of the regressed energy expenditure levels against the measured energy expenditure levels. A close tie can be seen between the reference and computed energy levels.

\section{Algorithm implementation}

Over the set of algorithms that were studied Chen's nonlinear algorithm performed best in comparison to the averaged energy expenditure values that were calculated. Figure 5 is a sample of the accelerometer regressed energy expenditure values and those measured by the CPET for each algorithm. The similarity between the regressed energy expenditure and the reference energy expenditure was determined by calculating the root mean squared error (RMSE) value between the datasets. For two signals, $x_{1}$, the reference, and $x_{2}$, the regressed signal, of the same length, $n$, the RMSE is given by (8). Two identical signals will give an RMSE $=0$.

$$
\mathrm{RMSE}=\sqrt{\frac{\sum_{i=1}^{n}\left(x_{1}(i)-x_{2}(i)\right)^{2}}{n}}
$$

The list of RMSE values associated with each algorithm can be found in Table IV.

It is not entirely surprising that Chen's linear and nonlinear algorithms performed so well because the algorithms are finely tuned to work for a person of a well defined stature, weight, gender etc. The fact that the nonlinear algorithm performance is best suggests accelerometer based energy expenditure regression may not be a linear process.

Bouten's algorithm performed relatively well considering the parameters of the equation were given 'as is' and were not dependent on mass, height, or gender of the subject. While the results of Chen's algorithms are expected to remain accurate with a growing signal database it is unsure how the results of Crouter and Bouten's algorithms will be affected.

Crouter's algorithm appeared to consistently overestimate the energy expenditure which contributed heavily to it performing poorest out of the algorithms investigated. However, the energy trace was smooth and did follow the trend of the metabolic response, see Figure 5(d).

\section{E. SHIMMER-domain implementation}

The implementation of the algorithms were then transferred to the SHIMMER domain. In order to validate the performance of the SHIMMER as a standalone and wireless platform for applications two SHIMMERs were worn on the same location of a body and the wearer was requested to walk at a comfortable walking pace. One SHIMMER was programmed to transmit real-time acceleration data to a computer, as was done in the data collection stage, while the second SHIMMER was programmed to regress the accelerations that were detected to energy expenditure measurements as described earlier. The acceleration data that was streamed to the computer was then analysed in Matlab and compared to the energy expenditure levels that were calculated on the second SHIMMER. The two datasets were found to be always within $2 \%$ of one another, suggesting that the SHIMMER is an ideal device for real time energy expenditure estimation.

The linear algorithm was first implemented due to the fact that it is a relatively straightforward algorithm to implement and that it performed well in comparison to the reference data points. Once the implementation was validated and verified Chen's second algorithm and Bouten's algorithm were likewise transferred to the SHIMMER-domain. The difference between the SHIMMER-domain and Matlab implementations were likewise within $2 \%$ of one another. This $2 \%$ discrepancy is due to the slightly different orientation and position of two SHIMMERs on the body for this experiment.

\section{Discussion AND CONCLUSIONS}

It is quite clear from the RMSE values presented in Table IV that accelerometers are a well suited instrument for estimating energy expenditure, in comparison to the gold standard, i.e. indirect calorimetry. Each of the algorithms investigated here have different assets (Bouten's algorithm works well with constant parameters over a wide set of users, Chen's algorithms follow the metabolic trends well due to the subject specific parameters of the equations, and Crouter's algorithm follows the trend of metabolic change well), and each also have aspects that might be improved upon.

Accelerometers can detect changes in motion, but where they fail in the question of energy expenditure estimation is in the enumeration of the effort required for movement. For example, an accelerometer will detect the motion of a moving arm, but if a weight was attached to the arm as it moves accelerometers cannot estimate the new effort required without foreknowledge of the weight.

The accuracy of all of the algorithms is subject to the reference their results are compared against. As the reference energy expenditure levels are not periodic and the time stamps are rounded to the nearest second some means of 


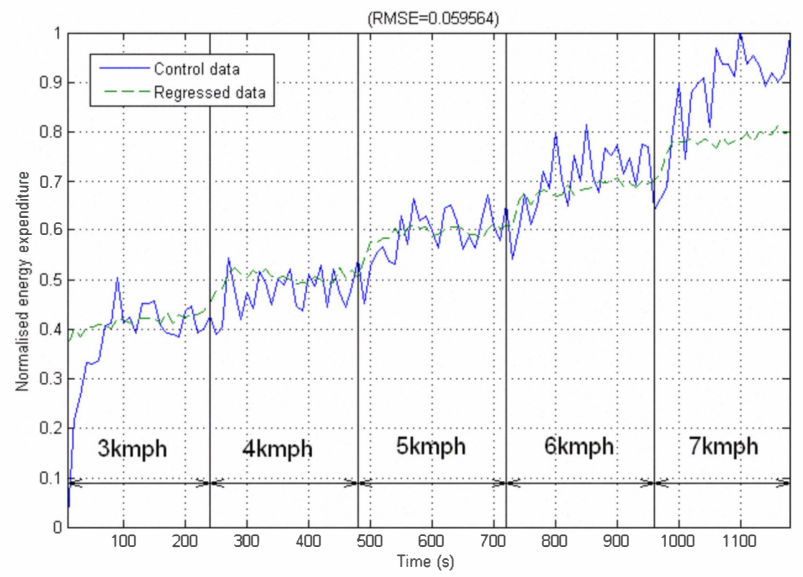

(a) Chen nonlinear

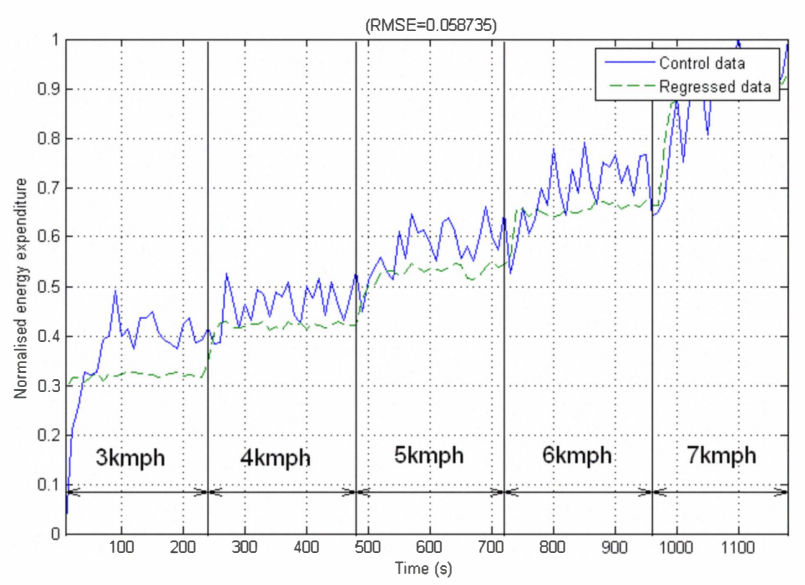

(c) Bouten

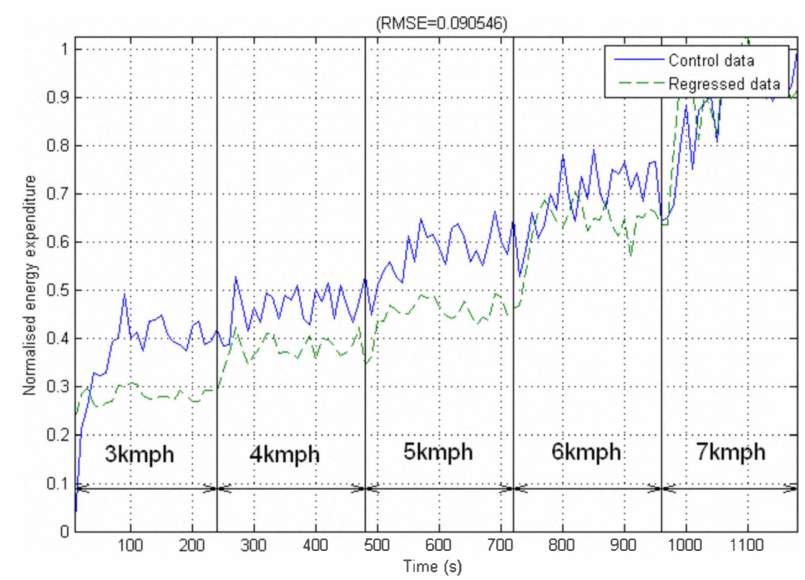

(b) Chen linear

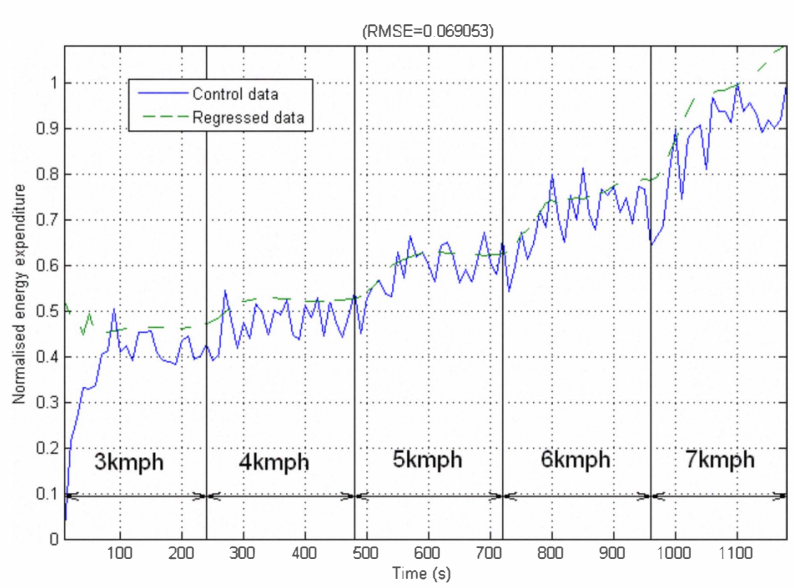

(d) Crouter

Figure 5. Sample plots for normailsed results of Bouten, Chen and Crouter's energy expenditure algorithms plotted with the reference levels

manipulation must be performed on the original reference data to coordinate its measurements with others. This process could potentially introduce inaccuracies to the reference data. As the method employed here involved averaging the breath data over an epoch a level of filtering was introduced to the reference data, which will reduce any noise that has been introduced, in effect a dynamic width moving average filter is being used.

Indeed, the reference energy levels can themselves be corrupted before any analysis is performed. This can happen on the treadmill through the subject yawning, coughing or even talking and while the subjects were requested to keep this to a minimum the possibility of artefacts siphoning through to the reference data must be accounted for. Measurement artefacts will accelerate, exaggerate or otherwise affect the reference values and the times they are measured. Lowpass filtering should remove most of these, and as this was performed by the epoch measurement-averaging algorithm discussed earlier, a significant portion of the artefacts were deemed to have been sufficiently reduced.

That Chen's algorithms provide excellent estimations of energy expenditure is a very strong indication that subjectspecific parameters in regression equations are one of the leading factors for accurate regression to energy expenditure values. The one shortcoming of the algorithm is that its output does not follow the trend of energy change when a change of pace is invoked. It is possible that this could be dealt with by utilising a moving average filter over the last number of algorithm energy levels.

Due to Chen's nonlinear algorithm resulting in the best correlations against the reference dataset the assumption of linearity between measured energy expenditure and acceleration may only be true only over a certain operation range. For these trials, however, the treadmill was set to move from 3 to $7 \mathrm{kmph}$. $7 \mathrm{kmph}$ is a very quick walk and many might feel more comfortable to jog at that pace. It may be more 
fitting to use $E E$ regression equations for running at these quicker paces, as the algorithms used may be out of their operating range.

The algorithms of Bouten and Crouter do not target the individual but rather were developed to accommodate the common user. This carries a convenient 'plug and play' feature to the algorithms (no setup required) but in counterpoint the accuracy of energy expenditure estimation is traded away, as can be seen seen by the results, with Crouter's more than Bouten's.

While the correlations between the reference and estimated energy expenditure levels are high (low RMSE) it is clear to see that a high variation exists between these two datasets. A number of factors could lead to this. As mentioned previously the metabolic response to an activity change does not occur instantaneously. Between the times of the initial change in motion and reaching of steady state by the body the difference between the,ation, a few percent of correct orientation, instantaneous change in regression levels and the rise of measured values will contribute to the noise in the plots below.

While the positioning and orientation of the devices was done with care, it cannot be guaranteed that the accelerometers were precisely vertical. Investigation into errors of orientation were done by Wang et al[11] and Bouten et al[12]. It was discovered that once the devices were 'close' to the correct orientation, within a few percent of a perfect positioning, the results were not badly affected. This positioning was accomplished by accurate fabrication of the straps that held the devices to the body. The devices themselves slotted tightly into a pouch limiting any vibrational motion. The straps were wide enough to reduce orientation errors to the point where they were unimportant.

This study shows that many accelerometer-based energy expenditure estimation algorithms can produce accurate results, and that a SHIMMER is an ideal platform on which they may be implemented.

\section{ACKNOWLEDGEMENTS}

The authors would like to extend their thanks to Adrian Burns, Intel Ireland, Dr John Bradley from the Department of Education, UCC, and Mr. Trevor Woods of the Human Performance Laboratory, Mardyke Arena for their assistance.

This research was funded by an enterprise partnership scheme between IRCSET (Irish Research Council for Science, Engineering \& Technology) and the TRIL Centre
(Technology Research for Independent Living), and Science Foundation Ireland (SFI/07/SRC/I1169).

\section{REFERENCES}

[1] KING, G. A., N. TORRES, C. POTTER, T. J. BROOKS, and K. J. COLEMAN. 'Comparison of Activity Monitors to Estimate Energy Cost of Treadmill Exercise.' Med. Sci. Sports Exerc., Vol. 36, No. 7, pp. 1244-1251, 2004.

[2] http://www.cosmed.it

[3] http://www.shimmer-research.com

[4] C. Bouten, K. Koekkoek, M. Verduin, R. Kodde, and J. Janssen, 'A triaxial accelerometer and portable data processing unit for the assessment of daily physical activity,' Biomedical Engineering, IEEE Transactions on, vol. 44, 1997, pp. 136-147.

[5] Jones, Andrew M. and Doust, Jonathan H.(1996) 'A 1\% treadmill grade most accurately reflects the energetic cost of outdoor running', Journal of Sports Sciences, 14:4, pages 321 327

[6] M Chatagnon1 and T Busso, 'Modelling of aerobic and anaerobic energy production during exhaustive exercise on a cycle ergometer,' European Journal of Applied Physiology, pages 755760

[7] Winter, Edward. 'Sport and Exercise Physiology Testing Guidelines.' New York: Routledge, 2007.

[8] C. Bouten and J. Janssen, 'Assessment of energy expenditure for physical activity using a triaxial accelerometer,' IEEE Transactions on Biomedical, vol. 44(3), pp. 136-147, 1997

[9] K.Y. Chen and M. Sun, 'Improving energy expenditure estimation by using a triaxial accelerometer,' J Appl Physiol, vol. 83, Dec. 1997, pp. 2112-2122.

[10] S.E. Crouter, K.G. Clowers, and D.R. Bassett, 'A novel method for using accelerometer data to predict energy expenditure,' Journal of Applied Physiology, vol. 100, Apr. 2006, pp. 1324-1331.

[11] L. Wang, S. Su, B. Celler, and E. Ambikairajah, 'Analysis of Orientation Error of Triaxial Accelerometers on the Assessment of Energy Expenditure,' Engineering in Medicine and Biology Society, 2005. IEEE-EMBS 2005. 27th Annual International Conference of the, 2005, pp. 3514-3517.

[12] C. Bouten and J. Jansse, 'Effects of placement and orientation of body-fixed accelerometers on the assessment of energy expenditure during walking,' Medical and Biological Engineering and Computing, 2006, 50-56 